\title{
Estudio in vitro de la alteración dimensional de impresiones con silicona por adición sometidas a desinfección
}

Hidalgo I, Balarezo A. Estudio in vitro de la alteración dimensional de impresiones con silicona por adición sometidas a desinfección. Rev Estomatol Herediana 2004;14(1-2) : 45 - 50.

\section{RESUMEN}

El propósito de este estudio fue evaluar la alteración dimensional en modelos de yeso tipo IV obtenidos de impresiones dentales con silicona por adición después de ser desinfectadas por inmersión durante 10 minutos con glutaraldehído al 2\% e hipoclorito de sodio al 1\%. Los modelos de trabajo fueron evaluados mediante una máquina de medición por coordenadas(con exactitud de $1 \mathrm{~mm}$ ). Se evaluaron cinco medidas: diámetro de la cara oclusal de la molar derecha, medida de distal a nivel del hombro a central de la cara oclusal de la molar derecha, medida desde el punto medio de la cara oclusal de canino a premolar, punto medio de la cara oclusal de premolar a molar izquierda y punto medio de cara oclusal de molar derecha a molar izquierda. Se encontraron diferencias estadísticamente significativas, para el grupo desinfectados por glutaraldehído al $2 \%$ comparadas con el modelo maestro. El grupo de hipoclorito de sodio al $1 \%$ no mostró diferencias estadísticamente significativas en los modelos de trabajo evaluados por lo que debe considerarse el desinfectante de primera elección para impresiones con silicona por adicción.

\section{Palabras claves: HIPOCLORITO DE SODIO / GLUTARAL / SILICONAS.}

In vitro study of dimensional alteration of addition reaction silicone dental impressions submitted to disinfection

ABSTRACT

The purpose of this study was to evaluate the dimensional alteration in type IV gypsum stone casts obtained from dental impressions with addition reaction silicone after disinfection by ten minutes immersion in $2 \%$ glutaraldehyde and in $1 \%$ sodium hypoclorite. The casts were evaluated with a coordinates measuring machine. Five measurements were evaluated: occlusal mesiodistal diameter of right molar, from distal shoulder to occlusal midpoint of right molar, from occlusal midpoint of right canine to left bicuspid, from occlusal midpoint of left bicuspid to left molar, from occlusal midpoint of right molar to left molar. There were statistically significant differences only for the glutaraldehyde disinfected group compared versus maste model. The $1 \%$ sodium hypoclorite group didn't show statistically significant differences in the casts for which reason we can consider it as a first choice disinfectant in the clinical practice.

Keywords : SODIUM HYPOCHLORITE / GLUTARAL / SILICONES.
Ivonne Hidalgo López ${ }^{1}$ Antonio Balarezo Razzeto ${ }^{1}$

'Docente del Departamento Académico de Clínica Estomatológica.

Facultad de Estomatología. Universidad Peruana Cayetano Heredia.

\section{Introducción}

En rehabilitación oral, una de las actividades comunes es la toma de impresiones dentales, que deben cumplir ciertos requisitos para garantizar el éxito final del tratamiento. Las impresiones no deben representar vehículos de transmisión de microorganismos que puedan generar contaminación cruzada entre pacientes y personal dental.

En la literatura se establece la fuerte influencia que ejercen la naturaleza del desinfectante, efecto del tiempo, técnica de desinfección utilizada y la concentración de los desinfectantes en los cambios dimensionales de las impresiones. Es necesario escoger adecuadamente el tipo y las condiciones en las que debe actuar el desinfectante te- niendo en cuenta el material de impresión utilizado.

Actualmente la silicona por adición representa el material de impresión con ventajas clínicas de gran alcance gracias a su excelente estabilidad dimensional. Esta propiedad implica la habilidad de un material de impresión de mantener precisión en sus medidas tridimensionales a lo largo del tiempo, en condiciones de humedad y temperatura determinadas.

Una forma de evaluar la estabilidad dimensional es a través del tiempo disponible para el vaciado. Cuanto mayor es el tiempo disponible para el vaciado más estable es el material. Una menor estabilidad dimensional deberá compensarse con el vaciado rápido de la impre- sión (1).

La estabilidad dimensional de la silicona por adición permite que los vaciados de las impresiones pueden postergarse por periodos largos debido a que no libera subproductos durante su polimerización $(2,3)$.

Al hacer la elección de la técnica de impresión, se debe considerar tiempo, costo y exactitud. La selección de una técnica específica depende de la experiencia y evaluación individual del paciente (4).

Se conocen tres técnicas de impresión para silicona por adición: técnica de impresión en dos tiempos, la impresión simultánea y la impresión monofásica. De acuerdo a la literatura se dice que la técnica de doble impresión es más 
precisa, debido a que la primera impresión realizada con el material denso hace el papel de una cubeta individual mucho más exacta, posteriormente el alivio de esta fase es vital para conseguir que el material fluido de la segunda fase de la impresión copie fielmente los detalles. Los modelos obtenidos por la técnica de doble impresión fueron más estables que las impresiones con la técnica de impresión única simultánea (5).

Después de realizada la impresión con el material y la técnica adecuada, el paso que sigue antes del vaciado de la impresión es la desinfección. Una adecuada interacción entre los agentes químicos de desinfección y los materiales de impresión trae como resultado modelos de trabajo libres de contaminación con microorganismos, sin sacrificar la exactitud y las dimensiones lineales de las impresiones dentales.

Hipoclorito de sodio: Las soluciones de hipoclorito de sodio son compuestos inorgánicos que pertenecen al grupo de los compuestos halogenados, los cuales tienen un efecto bactericida, en general, por su efecto oxidante (6). Todos los compuestos de cloro, actúan por una parte produciendo oxígeno y, por otro lado, combinándose con las proteínas bacterianas. La mayor parte de las bacterias son sensibles al cloro a concentraciones menores a una parte por millón, pero su actividad se dificulta por la presencia de materia orgánica (7).

Los compuestos de cloro son corrosivos, corroen el níquel y el acero cromado, el hierro y otros materiales oxidables. Las soluciones que exceden el $0.5 \%$ de Cloro disponible no deben ser usadas con frecuencia para la descontaminación de equipos de acero inoxidable de buena calidad. El contacto no debe durar más de 30 minutos.

Las soluciones deben de ser de manufacturación reciente y almacenadas en lugares protegidos del calor y de la luz. Las diluciones deben ser preparadas inmediatamente antes de su utilización. La descomposición rápida es un problema particular en las zonas de clima cálido (8).

Glutaraldehido: Las soluciones de glutaraldehído son compuestos orgánicos, del grupo de los aldehídos que tienen efecto sobre las bacterias. Se encuentra comúnmente disponible bajo la forma de una solución acuosa que necesita ser activada antes de su utilización, mediante la adición de sales de estaño a un $\mathrm{pH}$ alcalino, de esta forma adquiere poder esterilizante. Las soluciones al $2 \%$ no sólo destruye las formas vegetativas de las bacterias, sino también las esporas y los virus, como el de la hepatitis. La total inmersión de una estructura en una solución activada destruye las bacterias vegetativas, hongos y virus, generalmente con un tiempo promedio de 10 minutos. Se requiere de una inmersión de 10 horas para destruir las esporas (7).

Las soluciones de glutaraldehído son costosas, irritantes y emanan un vapor tóxico que los operadores deben de evitar. El contacto del líquido con la piel debe ser igualmente evitado (8).

La desinfección por inmersión es la más común y de más confianza debido al máximo contacto que se consigue entre el desinfectante, el material de impresión y la superficie de la cubeta. En esta técnica la estabilidad dimensional de la impresión es el factor crítico, en especial en materiales de naturaleza muy hidrofílica, como los hidrocoloides (agar-agar, alginatos) (9).

Los lineamientos de la Asociación Dental Americana (ADA) en cuanto al control de la infección de las impresiones recomienda la desinfección por inmersión. Con relación a la estabilidad dimensional, la técnica que ha producido una mayor controversia es la inmersión en solución desinfectante. Esta técnica es recomendada por la ADA para ser utilizada preferentemente en elastómeros por su mayor eficacia antiséptica y por ser capaz de compensar la contracción de polimerización de estos materiales mejorando la precisión, sin embargo, ha sido de gran controversia su utilización en poliéteres o hidrocoloides por su naturaleza altamente hidrofílica (10).

Aun así, esta técnica puede ser utilizada sobre cualquier tipo de material de impresión, siempre y cuando se adapte al antiséptico y el tiempo de inmersión a cada tipo de material $(9,11-13)$.

En las superficies de impresiones de polivinil siloxano (14) se retienen signi- ficativa-mente menos microorganismos que en otros materiales. Los polivinil siloxanos disponen de una tolerancia excelente a la inmersión en hipoclo-rito de sodio (15-20), glutaraldehído $(15,19)$, iodóforo $(15,18,19)$ y fenoles $(15,16)$.

La eficacia de un desinfectante va a depender de su concentración, tiempo de exposición, tipo de concentración de gérmenes y de la cantidad de residuos presentes (8). Se recomienda sumergir las impresiones en las soluciones diluidas durante un tiempo promedio de 10 minutos, excepto en el dióxido de cloro, en el que se sumergen sólo durante tres minutos. Los troqueles de yeso piedra de gran resistencia obtenidos partir de estas impresiones tienen una precisión excelente cuando se usan siliconas de adición y aceptable con los polisulfuros, pero inaceptable cuando se usan poliéteres. Debido a ello, no se recomienda desinfectar por inmersión a las impresiones de poliéteres, salvo durante períodos muy breves (2-3 minutos) con los desinfectantes clorados (21-23).

El efecto de tiempo de desinfección en los cambios dimensionales de las impresiones dentales es muy importante y está directamente relacionado con la efectividad del desinfectante, es decir que el tiempo suministrado debe ser el necesario para eliminar los microorganismos y a la vez no alterar dimensionalmente las impresiones. Delgado et al. (24) han mencionado que el glutaraldehído al $2 \%$, o el hipoclorito de sodio al $1 \%$ en una inmersión de 10 minutos son altamente efectivos para desinfectar las impresiones de silicona por adición (24).

Considerando que los cambios dimensionales de las impresiones después de la desinfección puedan afectar la exactitud de los modelos de yeso, y esto consecuentemente afectará enormemente la adaptación de las restauraciones de prótesis fijas y removibles, el propósito del presente estudio fue evaluar dicha alteración en modelos de trabajo obtenidos de impresiones con silicona por adición al ser sometidos por inmersión de 10 minutos a dos agentes desinfectantes: glutaraldehido al 2\% y a hipoclorito de sodio al $1 \%$, agentes desinfectantes comúnmente utilizados en la actualidad. 


\section{Material y método Impresión del modelo maestro}

Se tomaron 45 impresiones del modelo maestro, el cual se confeccionó en acero inoxidable (Fig. 1), y simula una arcada mandibular, contiene cuatro pilares cónicos con preparaciones para prótesis fija. Las impresiones se tomaron con la ayuda de una cubeta metálica de acero inoxidable y se utilizó la técnica de doble impresión. Se dispensaron cantidades iguales de silicona por adición masa y fluida (Elite H-D Zhermack $^{\circledR}$ ) siguiendo las indicaciones del fabricante. Para uniformizar las capas de silicona por adición (masa y fluida) se utilizó una capa de alivio confeccionada con resina acrílica de $2 \mathrm{~mm}$ de grosor sobre el modelo maestro, que tenía las huellas de los surcos de escape. La superficie del modelo se presentaba altamente pulida para evitar desgarros al momento de retirar la impresión. Dichas impresiones se realizaron a una presión constante de $12 \mathrm{Kg} / \mathrm{cm}^{2}$ y con un tiempo de asentamiento de cinco minutos, tiempo suficiente para la polimerización. La prensa aplicó una presión constante para uniformizar esta condición en todos los casos asegurándose siempre un encaje perfecto de la cubeta con la base del modelo maestro. Después de retirar la cubeta y la capa de alivio en dirección axial, se tomo la segunda fase de la impresión con silicona fluida en la forma de cartuchos y con ayuda de una jeringa de automezclado, de esta forma se consiguió una mezcla uniforme y ausencia de burbujas. Se colocó nuevamente bajo la prensa, y en las condiciones descritas, se tomaron y retiraron del modelo metálico la segunda impresión (Fig. 2).

Luego de ésto, se procedió a asignar un grupo aleatoriamente de acuerdo al desinfectante a utilizar:

Grupo hipoclorito de sodio.- Se colocó en inmersión por 10 minutos en soluciones de hipoclorito de sodio al $1 \%$ según protocolo de desinfección (25)y finalmente se enjuagó con agua por 10 segundos.

Grupo glutaraldehído.- Se colocó en inmersión por 10 minutos en soluciones de glutaraldehído al 2\% según protocolo de desinfección (25) y finalmente se enjuagó con agua por 10 segundos. Grupo control.- Sólo se enjuagó con agua por 10 segundos.

El exceso de líquido se eliminaba con una corriente de aire proveniente de la jeringa triple. Dos horas después (según indicaciones del fabricante) se vaciaron las impresiones con yeso tipo IV marca Velmix $^{\circledR}$, en una cantidad constante para todos los casos, el peso se determinó con ayuda de una balanza de semiprecisión, la cantidad de agua a $5^{\circ} \mathrm{C}$ se determinó con una pipeta graduada. El espatulado fue primero mecánico y luego al vacío durante 20 segundos. Se esperó una hora para retirar los modelos después del vaciado con yeso.

\section{Medición de los modelos de yeso y del modelo maestro}

Las medidas fueron tomadas con la máquina de medición por coordenadas modelo Beyond 700/900 Mitutoyo Corporation $^{\circledR}$ (Fig. 3). Esta máquina de precisión toma medidas en tres dimensiones en los ejes X, Y, Z con exactitud de 1 micra y los digitaliza en la computadora con ayuda del Software “Scanpack” versión 3.1. Estas medidas son registradas cuando el cabezal medidor toca la superficie del modelo de yeso con la ayuda de un sensor de contacto superficial o palpador, brindando así mediciones de alta precisión.

Las mediciones fueron realizadas en el Laboratorio del CETAM de la Pontificia Universidad Católica del Perú, en condiciones ambientales de $20^{\circ} \mathrm{C}$ de temperatura y a humedad de 64\%. Se registraron cinco tipos de mediciones (Fig. 4) en los 45 modelos de yeso y el modelo maestro y posteriormente se realizaron las comparaciones. Todas las medidas fueron realizadas tres veces, para controlar el error humano, por lo cual se obtuvieron un total de 690 medidas.

Se realizó un análisis univariado de la alteración dimensional para cada grupo a través de los promedios y desviación estándar y se evaluó la normalidad de los datos utilizando la prueba estadística de Kolmogorov- Smirnow. Para la comparación de datos de los tres grupos contra una norma(modelo maestro) se utilizó la prueba estadística t de Student.

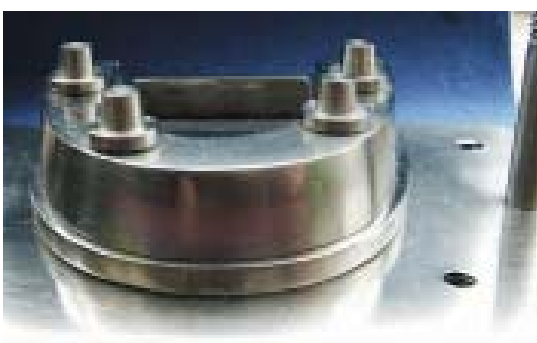

Fig. 1. Modelo metálico maestro.

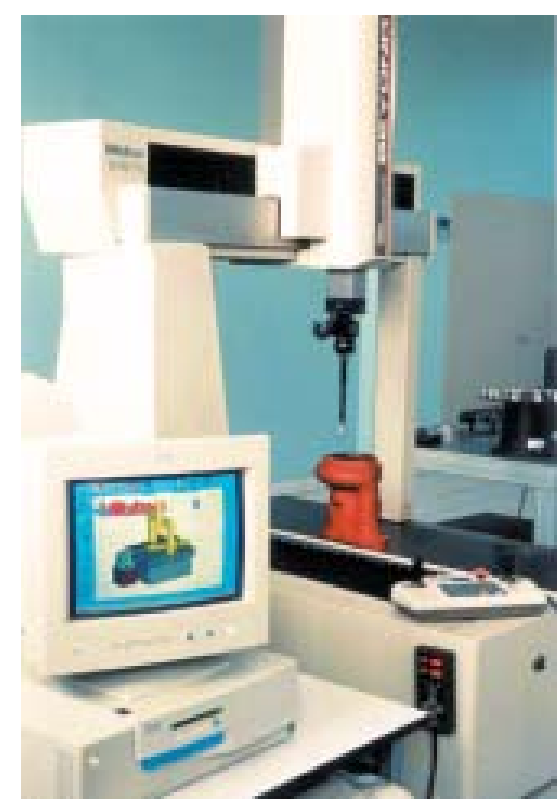

Fig. 3. Máquina de medición por coordenadas (Beyond 700/900 Mitutoyo Corporation).

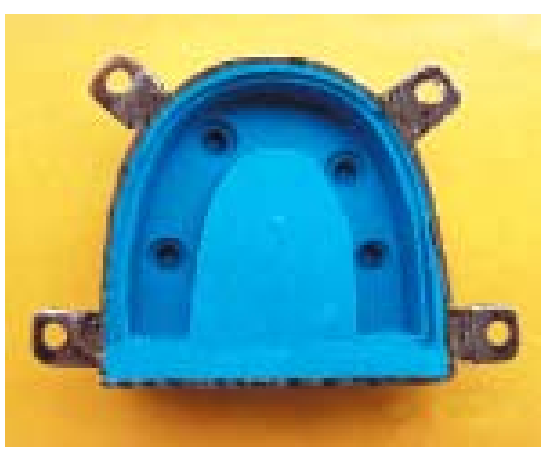

Fig. 2. Segunda impresión.

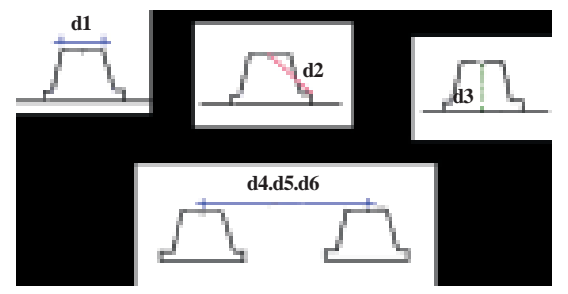

Fig. 4. Cinco tipos de mediciones. 


\section{Resultados}

Los resultados de las cinco mediciones realizadas a cada una de las 45 muestras del estudio, demuestran que el grupo sin desinfección, es el que más se acerca a las medidas del modelo maestro, seguido del grupo hipoclorito de sodio (Tabla 1).

En el grupo de Glutaraldehído se observaron diferencias estadísticamente significativas para la medida d1 (diámetro mesiodistal de la pieza molar derecha), y la medida d4 ( distancia desde el punto medio de cara oclusal de premolar a molar izquierda) (Tabla 2).

\section{Discusión}

Para realizar un buen trabajo protésico, es indispensable que la impresión deba ser precisa en la reproducción de los detalles de la preparación dentaria. La utilización de siliconas de adición me- jora la posibilidad de conseguir impresiones con dichas características $(6,26)$.

Actualmente existe continua preocupación por el peligro de la contaminación cruzada potencial de los microorganismos por intermedio de las impresiones dentales. Este material de impresión se expone al contacto con saliva y sangre, convirtiéndose en un medio de contaminación cruzada $(27,28)$. Ante esta situación, se requiere de un protocolo de desinfección que a su vez asegure la estabilidad dimensional de las siliconas de adición $(11,29)$.

En esta investigación no se encontraron diferencias estadísticamente significativas para el grupo control y para el grupo de hipoclorito de sodio al $1 \%$ después de la inmersión por 10 minutos. Estos datos concuerdan con los hallados por varios autores, para quienes las variaciones medidas después del tratamiento con hipoclorito de sodio al $0.5 \%$ no fueron significativas $(10,11,28)$.

Herrera y Merchant (15) evaluaron también el uso del hipoclorito de sodio con $1 \%$ de concentración, y tampoco encontraron diferencias estadísticamente significativas, recomendando el uso de este desinfectante por ser a la vez más económico. Matyas et al. (16) estudiaron el efecto del hipoclorito de sodio al 1\% en las impresiones de silicona por adición, después de su inmersión por 10 minutos, encontraron que no producían ninguna variación dimensional en las mediciones anteroposteriores y arco cruzado de una arcada mandibular y en la medida mesiodistal de una pieza con preparación para prótesis fija unitaria. La estabilidad se consigue debido a la baja concentración utilizada, ya que el cloro es un elemento altamente reactivo, actúa liberando oxígeno, y a concentra-

Tabla 1. Media y Desviación Estándar de los grupos de evaluación ( en milímetros )

\begin{tabular}{|c|c|c|c|c|c|c|c|c|c|c|c|}
\hline \multirow[t]{2}{*}{ Grupo } & \multirow[t]{2}{*}{$\begin{array}{l}\mathrm{N}^{0} \text { de } \\
\text { muestra }\end{array}$} & \multicolumn{2}{|c|}{ d1 } & \multicolumn{2}{|c|}{ d2 } & \multicolumn{2}{|c|}{ d3 } & \multicolumn{2}{|c|}{ d4 } & \multicolumn{2}{|c|}{ d5 } \\
\hline & & Media & D.S. & Media & D.S. & Media & D.S. & Media & D.S. & Media & D.S. \\
\hline $\begin{array}{l}\text { Hipoclorito de sodio } \\
\text { (A) }\end{array}$ & 15 & 5.271 & 0.0297 & 6.214 & 0.1228 & 30.567 & 0.0618 & 20.052 & 0.0521 & 46.015 & 0.052 \\
\hline $\begin{array}{c}\text { Glutaraldehído } \\
\text { (B) }\end{array}$ & 15 & 5.213 & 0.07 & 6.313 & 0.2206 & 30.592 & 0.041 & 20.069 & 0.0561 & 46.047 & 0.113 \\
\hline $\begin{array}{l}\text { Sin desinfección } \\
\text { (C) }\end{array}$ & 15 & 5.256 & 0.019 & 6.228 & 0.0239 & 30.585 & 0.0227 & 20.028 & 0.0167 & 45.995 & 0.0158 \\
\hline Modelo maestro & 1 & 5.2 & & 6.2 & & 30 & 596 & & & 45 & \\
\hline
\end{tabular}

d1 = La medida de la cara oclusal (diámetro mesio-distal) de la molar derecha

$\mathbf{d 2}=$ La medida de distal (desde el hombro) a central de la cara oclusal de la molar derecha

d3 = La medida desde punto medio de cara oclusal de canino a premolar

d4 = Punto medio de cara oclusal de premolar a la molar izquierda

d5 = Punto medio de cara oclusal de la molar derecha a la molar iaquierda

Tabla 2. Prueba t de student para las medidas de los tres grupos comparados con el modelo maestro

\begin{tabular}{|c|c|c|c|c|c|c|c|c|c|c|c|}
\hline \multirow[t]{2}{*}{ Grupo } & \multirow[t]{2}{*}{ gl } & \multicolumn{2}{|c|}{$\begin{array}{l}\text { Valor de prueba = } 5.26 \\
\text { d1 }\end{array}$} & \multicolumn{2}{|c|}{$\begin{array}{c}\text { Valor de prueba }=6.235 \\
\text { d2 }\end{array}$} & \multicolumn{2}{|c|}{$\begin{array}{c}\text { Valor de prueba }=30.596 \\
\text { d3 }\end{array}$} & \multicolumn{2}{|c|}{$\begin{array}{c}\text { Valor de prueba }=20.024 \\
\text { d4 }\end{array}$} & \multicolumn{2}{|c|}{$\begin{array}{c}\text { Valor de prueba }=45.99 \\
\text { d5 }\end{array}$} \\
\hline & & t & $\mathbf{p}$ & t & $\bar{p}$ & t & $\bar{p}$ & t & $\bar{p}$ & $\mathbf{t}$ & $\bar{p}$ \\
\hline $\begin{array}{l}\text { Hipoclorito de sodio } \\
\text { (A) }\end{array}$ & 14 & 1.39 & 0.186 & -0.66 & 0.519 & -1.80 & 0.093 & 2.10 & 0.053 & 1.88 & 0.080 \\
\hline $\begin{array}{l}\text { Glutaraldehído } \\
\text { (B) }\end{array}$ & 14 & -2.59 & $0.021^{*}$ & 1.37 & 0.193 & -0.41 & 0.684 & 3.16 & $0.007^{*}$ & 1.76 & 0.100 \\
\hline $\begin{array}{l}\text { Sin desinfección } \\
\text { (C) }\end{array}$ & 14 & -0.97 & 0.351 & 1.08 & 0.3 & -1.85 & 0.085 & 0.95 & 0.354 & 1.21 & 0.246 \\
\hline
\end{tabular}

gl $=\quad$ Gardos de libertad

$*$ = Diferencia estadísticamente significativa con p 0.05

$\mathbf{d} 2$ = La medida de distal (desde el hombro) a central de la cara oclusal de la molar $\leq$ derecha

d3 = La medida desde punto medio de cara oclusal de canino a premolar

d4 = Punto medio de cara oclusal de premolar a la molar izquierda

$\mathbf{d 5}=$ Punto medio de cara oclusal de la molar derecha a la molar izquierda 
ciones mayores podría reaccionar alterando el material de impresión, produciendo una expansión adicional del yeso (23).

Esto se evidencia en el trabajo de Thouati et al. (9) para quienes las variaciones producidas después de la desinfección con hipoclorito de sodio al $5.25 \%$ en 30 minutos de inmersión fueron significativas cuando las compararon con el grupo control que consistió en impresiones sin desinfección.

Estos hallazgos indican que impresiones desinfectadas con hipoclorito de sodio tienen poca estabilidad cuando se trabajan en tiempos largos y a concentraciones elevadas. Así, en la investigación realizada por Merchant (30), desinfectó impresiones de silicona por adición con igual tiempo de inmersión que el trabajo de Thouati pero con una concentración del 1\% y sus resultados mostraron que no se presentaron diferencias estadísticamente significativas.

En nuestro estudio se encontraron diferencias estadísticamente significativas para el diámetro de la cara oclusal en las impresiones desinfectadas con glutaraldehído al $2 \%$ por 10 minutos de inmersión. La medida del modelo metálico fue de $5.26 \mathrm{~mm}$, las medidas del grupo de hipoclorito de sodio al $1 \%$ presentaron un promedio de $5.271 \mathrm{~mm}$, el grupo sin desinfección con un promedio de $5.256 \mathrm{~mm}$ y el grupo de glutaraldehído al 2\% con un promedio de $5.213 \mathrm{~mm}$ y con una diferencia negativa con respecto a la medida del modelo metálico de $47 \mu \mathrm{m}$.

Estos resultados son similares a los encontrados por Lepe y Johnson (10) quienes también estudiaron el comportamiento de las impresiones de silicona de adición frente al glutaraldehído al 2\%, en donde encontraron diferencias estadísticamente significativas para las evaluaciones en sentido mesiodistal, resultando al igual que en nuestro estudio, con valores menores que el modelo maestro.

Esta diferencia negativa en sentido mesiodistal encontrada en el grupo de glutaraldehído indica que se elaborarán modelos más pequeños que el original, esto tendrá importancia en el procedimiento de las prótesis fija debido a que se produciría colados muy pequeños o muy ajustados, si es que no se puede compensar adecuadamente con el espaciador de modelos de yeso o con la expansión del yeso de revestimiento.

La especificación $\mathrm{N}^{\circ} 8$ de la ADA, menciona que se permite un desajuste (una diferencia positiva) del orden de los 30- 50 ìm en las regiones correspondientes al tercio medio y oclusal/incisal, ya que este espacio interno permitirá la ubicación de la película de cemento. Mientras que Lepe y Johnson (10) señalan que los cambios dimensionales permitidos en sentido antero posterior y mesiodistal presentan un rango desde 24 a 37 ìm, esta medida aproximadamente se compara con dos capas de espaciador y puede ser compensado.

Por todo esto, es conveniente que, las prótesis fijas deben presentar dimensiones mayores que los propios dientes, creando simultáneamente espacios internos necesarios para acomodar la película de cemento utilizado para fijación definitiva, sin por eso perder las características de retención (31, 32).

Otra medida que mostró diferencias estadísticamente significativas fue encontrada en el grupo de comparación d4 (distancia entre el punto medio de cara oclusal de premolar a molar izquierda) para las impresiones desinfectadas por glutaraldehído al $2 \%$ por 10 minutos de inmersión. La medida del modelo metálico fue de $20.024 \mathrm{~mm}$, el grupo de hipoclorito de sodio al 1\% presentó un promedio de $20.052 \mathrm{~mm}$; el grupo sin desinfección con un promedio de 20.028 mm y el grupo de glutaraldehído al 2\% con un promedio de $20.069 \mathrm{~mm}$ y con un aumento con respecto al modelo metálico de $45 \mu \mathrm{m}$.

Estos resultados no están de acuerdo con los de Herrera y Merchant (15) quienes compararon estabilidad dimensional de las impresiones de silicona de adición sometidas a inmersión en glutaraldehído al $0.13 \%$ y al 2\%, en los cuales no encontraron alteraciones dimensionales en modelos de yeso provenientes de dichas impresiones. Cabe resaltar que estas mediciones fueron tomadas con un Calibrador digital electrónico, cuya exactitud es menor a la de una máquina de medición por coordenadas digitalizadas por computadora y con exactitud de una micra. Las diferencias podrán deberse a la metodología empleada.

También Langenwalter (28) encon- tró que la desinfección con glutaraldehído al $2 \%$ no presenta diferencias estadísticamente significativas, pero aun así el hipoclorito mostró variaciones menores comparadas con el glutaraldehído.

Otra situación similar se presenta con el estudio desarrollado por Matyas et al. (16) para los cuales, si bien es cierto el glutaraldehído al 2\% en inmersión por 10 minutos, y medido con Microscopio de medición con exactitud de una micra no presentó alteraciones estadísticamente significativas, no produce mejores resultados que los conseguidos con el hipoclorito de sodio al $1 \%$.

Johnson et al. (12) también evaluaron el uso del glutaraldehído al $2 \%$ en 10 minutos de inmersión, frente a otros desinfectantes tales como: iodoforo, glutaraldehído fenol, aunque no tenía diferencias estadísticamente significativas, el glutaraldehído fue el desinfectante que más varió en comparación a los demás para las medidas bucolinguales o mesiodistales.

En otra investigación realizada por Kern et al. (33) desinfectaron las impresiones de silicona por adición con glutaraldehído al 1.1\%, y en la evaluación anteroposterior encontraron un porcentaje de variación menor al $0.1 \%$, esto puede deberse a la baja concentración del desinfectante.

Las alteraciones dimensionales halladas en nuestro estudio corresponden al sentido anteroposterior porque pertenecen a un mismo cuadrante. Estos cambios en sentido anteroposterior pueden deberse a la naturaleza química del desinfectante, debido a que la superficie del material de impresión tiene una afinidad con el surfactante presente en el desinfectante incrementando la rugosidad del material hasta el punto que afecta la humectabilidad del mismo, como lo demostraron los estudios de Pratten y Cavey (29).

Las alteraciones en el sentido antero posterior juegan un rol muy importante en la fabricación de prótesis fija y removible, ya que se altera la distancia entre dos pilares contiguos, impidiendo el buen asentamiento del puente. Sin embargo se dice que en prótesis removible esto puede resultar beneficioso debido a la contracción que sufren la estructura metálica de cromo-cobalto y que la expansión térmica del yeso de 
revestimiento por si sólo no puede compensar $(10,34)$.

Se puede inferir que la desinfección con hipoclorito de sodio no afecta el material de impresión, siempre que la concentración utilizada no sea elevada. Por esta razón se recomienda el uso del hipoclorito de sodio al 1\% en inmersión por 10 minutos como desinfectante de impresiones con silicona por adición, debido a su estabilidad después de la desinfección y su bajo costo.

\section{Referencias bibliográficas}

1. Vega del Barrio JM. Materiales en Odontología: Fundamentos Biológicos, Clínicos, Biofísicos y Fisicoquímicos. 1ra Ed. Madrid: Ediciones Avances Medico-Dentales S.L, 1996: 254.

2. Ciesco JH. Comparison of elastomeric impression materials used in fixed prosthodontics. J Prosthet Dent 1981; 45: 89.

3. Mezzomo E. Rehabilitación Oral para el Clínico. 2da Ed. Sao Paulo: Santos, 1997: 392.

4. Malone W. Tylman's teoría y practica en prostodoncia fija. 8va Ed. Caracas $(\mathrm{Vn})$ : Actualidades medico odontologicas latinoamerica, 1991:237.

5. Henry P, Harnist D. Dimensional stability and accuracy of rubber impresión materials. Aust Dent J 1974; 19(3): 162-66.

6. Eames WB, Wallace SW, Suway NB: Accuracy and dimensional stability of elastomeric impression materials. J Prosthet Dent 1979; 42: 159-162.

7. Setcos JC, Huizing K L, Palenik CJ. Antimicrobial abilities of a disinfectant- containing gypsum (tipe IV) stone (Abstract $\mathrm{N}^{\circ}$ 2080). J Dent Res 1991; 69: 308.

8. WHO Technical report series 512, Viral Hepatitis 1973.

9. Thouati A, Deveaux E, Iost A, Behin P. Dimensional stability of seven elastomeric impresion materials inmersed in disinfectans. J Prosthetic Dent 1996; 76 (1): 8-14.

10. Lepe X. Johnson G. Accuracy of polyether and addition silicone after long-term immersion disinffection $\mathrm{J}$ Prosthet Dent 1997; 78: 145 - 9.

11. Ríos MP, Morgano SM, Stein RS,
Rose L. Effects of chemical disinfectant solutions on the stability and accuracy of the dental impression complex. J Prosth. Dent 1996; 76 (4): 356-62.

12. Johnson GH, Chellis KD, Gordon GE, Lepe X. Dimensional stability and detail reproduction of irreversible hydrocolloid and elastomeric impressions disinfected by inmersion.J Prosthet Dent 1998;79(4):446-53.

13. Milian MA, Silvestre FJ. Esterilización y desinfección en Odontología. En: Esplugues J, Morcillo EJ, AndrésTrelles F. Farmacología en Clínica Dental. Barcelona: Ed. Prous JR, 1993:251-65.

14. Mansfield S, White J. Antimicrobial effects from incorporation of disinfectants into gypsum casts. Int $\mathrm{J}$ Prostodont 1991; 4(2): 180-85.

15. Herrera SP, Merchant VA. Dimensional Stability of dental impressions after inmersion disinfection. Am Dent Assoc. 1996; 113: 419 - 22.

16. Matyas J, Dao N, Caputo AA. Effects of disinfectants on dimensional accuracy of impression materials. J Prosthet Dent 1991; 64: 25-31.

17. Drennon DG, Johson GH, Powell GL. The accuracy and efficacy of disinfection by spray atomization. On elastomeric impressions J Prosthet Dent 1989;62:468-75.

18. Merchant VA. Prosthodontic and infection control - it's a whole new ball game. (Abstract) Calif Dent Assoc 1989;17:49-53.

19. Tebrock O, Engelmeier R. Mananging dental impressions and cast of patients with comunicable disease. Gen Dent 1989;37(6): 490-95.

20. Minagi S, Yano N, Yoshida K, Tsuru H. Prevention of an acquiered inmunodeficiency syndrome and hepatitis impressions materials. J Prosthet Dent 1987; 58: 462 -5.

21. Infection control recommendations for the dental office and the dental laboratory: council on dental practice council on dental therapeutics. J Am Dent Assoc 1988; 116: 241 - 8.

22. Owen CP, Goolam R. Disinfection of impressions materials to prevent viral cross contamination:a review and a protocol Int.J.Prosthodont 1933; 6: 480 - 94.

23. Pumarola A. Microbiología y Parasi- tología Médica.2 da Ed México DF: Salvat Editores, 1984: 111-115.

24. Delgado W, Flores G, Vives V. Control de las infecciones transmisibles en la Práctica Odontológica. Manual de Procedimientos. 1ra. Ed. Lima: Diseño Total, 1995.

25. Lorren R, Salter D. The contact angles of die stone on impression materials. J Prosthet Dent 1976; 36(2): 176-80.

26. Chorres JE. Evaluación de la alteración dimensional de troqueles en función del tiempo de ubicación del pin en el yeso (Tesis) Sao Paulo: Universidad de Sao Paulo,2001. 97 págs.

27. Pegoraro L. Prótesis Fija. $1^{\text {ra }}$ Ed. Sao Paulo: Editora Artes Médicas Ltda,2001: 225.

28. Langenwalter EM. The dimensional stability of elastomeric impression materials following disinfection. $\mathrm{J}$ Prostheth Dent 1990; 63:270-6.

29. Pratten DH, Covey DA. Effect of disinfectant solutions on the wet ability of elastomeric impression materials. J Prosthet Dent 1990; 63: 223 7.

30. Merchant V. Preliminary investigation of a method for disinfection of dental impressions. J Prosthet Dent 1984; 52(6): 877-79

31. Kess R, Combe E. Effect of surface treatments on the wettability of vinylpolysiloxane impression materials. J Prosthet Dent 2000;83:98102.

32. Abbate MF. Comparison of the marginal fit of various ceramic crown systems. J Prosthet Dent 1989; 61:527-31.

33. Kern M, Rather RM, Strub Jr. Three dimensional investigation of the accuracy of impressions materials after disinfection. J Prosthet Dent 1993; 70: 449 - 56.

34. Dykema RW. Modern practice in removable prosthodontics. 1st ed. Philadelphia : WB Saunders; 1969: 256.
Dirección de correspondencia
Ivonne Hidalgo López
El Galeón 535 La Castellana
Lima 33 - Perú
271-2081 991-6728
ivonne_hl@hotmail.com 